\title{
Precise Control of Pt Particle Size for Surface Structure-Reaction Activity Relationship
}

\author{
Reem Al-Shareef, ${ }^{\dagger}$ Moussab Harb, ${ }^{*},{ }^{\dagger}$ Youssef Saih, ${ }^{\dagger}$ Samy Ould-Chikh, ${ }^{\dagger}$ ' Dalaver H. Anjum, ${ }^{\ddagger}$ \\ Jean-Pierre Candy, ${ }^{\dagger}$ and Jean-Marie Basset ${ }^{*}{ }^{\dagger \oplus}$
}

†KAUST Catalysis Center (KCC), Physical Sciences and Engineering Division (PSE), King Abdullah University of Science and Technology (KAUST), Thuwal 23955-6900, Saudi Arabia

${ }^{\ddagger}$ Imaging and Characterization Lab, King Abdullah University of Science and Technology (KAUST), Thuwal 23955, Saudi Arabia

Supporting Information

\begin{abstract}
The use of surface organometallic chemistry on metal (SOMC/M) allows the controlled and stepwise variation of the platinum particle size in $\mathrm{Pt} / \mathrm{SiO}_{2}$ catalysts. This $\mathrm{SOMC} / \mathrm{M}$ method is possible thanks to the better affinity of most organometallic compounds with the surface of zerovalent metal particles covered with hydrogen than their support. In this paper, $\mathrm{Pt}(\mathrm{acac})_{2}$ was used as the organometallic precursor, silica as a support, and then hydrogen to reduce the adsorbed organometallic layer on top of the starting Pt nanoparticle. We partially succeeded in adding one Pt layer with a stepwise particle size increase of around $0.6 \mathrm{~nm}$ when going from the first $(1 \mathrm{G})$ to the second $(2 \mathrm{G})$ refilling

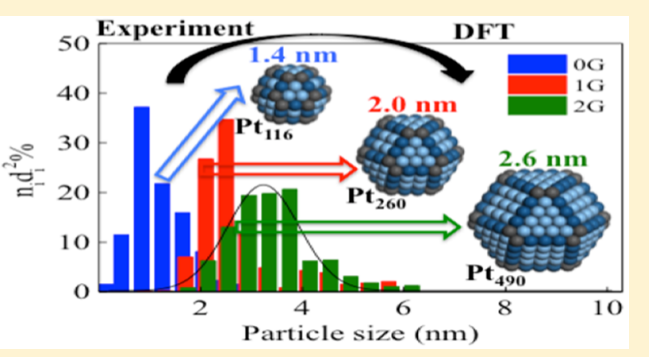
run, as obtained from TEM and $\mathrm{H}_{2}$ chemisorption analysis and then confirmed by DFT calculations. The metal loading could be kept at a very low level $(<1-2 \mathrm{wt} \%)$, which is relevant for catalytic applications. The particle size distribution remained relatively narrow even after two refilling runs, allowing more precise relationships between particle size and catalytic properties to be established. The $\mathrm{TOR}_{\mathrm{H}}$ (for hydrogenolysis) dramatically decreased, while $\mathrm{TOR}_{\mathrm{I}}$ (for skeletal isomerization) slightly increased with increasing the particles size. It is therefore suggested that hydrogenolysis might preferentially occur on low coordination surface platinum atoms (corners and edges), while isomerization occurs mostly on the facets.
\end{abstract}

\section{INTRODUCTION}

Metal particles supported on oxides are widely used as catalysts for several industrial catalytic processes. ${ }^{1,2}$ The catalytic properties of supported metals are determined by a number of highly connected parameters, mainly their size, shape, and specific crystallographic positions. The relationship between catalytic performance and size (or dispersion) of metallic nanoparticles is currently a hot subject in the field of catalysis by metals. ${ }^{3-10}$ In general, without considering these parameters, a decrease in the size of metallic particles should lead to an increase of the reaction rate measured per gram of metal. This is often explained by an increase in the metal atoms that are available on the surface and act as active sites (classically called dispersion). For some reactions, in particular when the size of these particles is below $5 \mathrm{~nm}$, the activity per surface metal atom (commonly referred to as turnover frequency (TOF)) has been shown to be also dependent on the morphology of the nanoparticles. In this case, M. Boudart has defined the reaction as structure-sensitive. Such a reaction is the hydrogenolysis of hydrocarbons, a reaction where aliphatic hydrocarbons are split with hydrogen over noble metals. ${ }^{11}$

Many efforts have therefore been made to prepare a series of supported metallic catalysts of different particle sizes with size distributions as narrow as possible in order to find meaningful relationships between size and properties. One of the methods commonly used to vary this parameter is sintering of the metallic phase at different temperatures and under adequate atmospheres. ${ }^{12-14}$ Particle growth by sintering is, however, difficult to precisely control since it involves a number of competitive processes, like migration and collision of small particles and sublimation through the gas phase (e.g., with $\mathrm{Ni}$ and $\mathrm{CO}){ }^{15}$ which are more mobile than large ones and are progressively consumed by collisions with other particles in the surface. Sintering may cause a very rapid particle growth resulting in particle size distributions extending from a few to several tens of nanometers. ${ }^{12,16}$

Alternative methods have therefore been established in order to allow a better control of the particle size and shape by controlling the growth process. One of these methods is the surface organometallic chemistry on metal (SOMC/M). ${ }^{17-19}$ In the past, the latter has been successfully used for numerous cases to prepare in a controlled manner "monodisperse" supported metallic particles with the same shape.

Here, we have used the SOMC/M approach to deposit a monolayer of metal atoms selectively on the surface of the monometallic particle. To add one monolayer of $\mathrm{Pt}$ atoms at a time, we need to know the number of $\mathrm{Pt}$ atoms on the surface of the particle. From $\mathrm{H}_{2}$ chemisorption, and based on certain

Received: July 3, 2018

Revised: September 26, 2018

Published: September 27, 2018 
assumptions on the stoichiometry of the chemisorption process, it is easy to determine this number of surface platinum atoms $\left(\mathrm{Pt}_{\mathrm{s}}\right)$. Based on this value, we introduced the same amount of soluble $\mathrm{Pt}$ complexes to get a full coverage of the particle by the organometallic precursors, avoiding adsorption on the support.

In a typical SOMC/M protocol, $\mathrm{Pt}$ nanoparticles with controlled size (and shape) could be prepared by selecting an appropriate starting Pt-supported catalyst, which exhibits a sharp particle size distribution. The amount of exposed $\mathrm{Pt}$ atoms is easily determined using $\mathrm{H}_{2}$ chemisorption, and the required amount of a suitable $\mathrm{Pt}$ organometallic precursor (e.g., in this case $\mathrm{Pt}(\mathrm{acac})_{2}$ ) can be deposited and subsequently decomposed using hydrogen, under adequate operating conditions, on the surface of the starting metal nanoparticle to form a new surface layer. In the following, the starting Pt catalyst will be named "host" (0G), and the obtained sample after reaction with $\mathrm{Pt}(\mathrm{acac})_{2}$ will be called first-generation (1G) catalyst. The same procedure will be applied to the first generation to yield a second-generation $(2 \mathrm{G})$ catalyst and can be further repeated as often as required to grow the desired number of layers.

The effect of the metallic dispersion on their intrinsic activity has been previously reported for the hydrogenolysis of ethane over $\mathrm{Ni} / \mathrm{SiO}_{2},{ }^{11}$ for the hydrogenolysis of neopentane on $\mathrm{Rh} / \gamma-\mathrm{Al}_{2} \mathrm{O}_{3},{ }^{20}$ and for cyclohexane on $\mathrm{Ir} / \mathrm{SiO}_{2}{ }^{21}{ }^{21}$ To the best of our knowledge, a consistent study of the effect of particle size on the isobutane hydrogenolysis and isomerization using Pt catalysts supported on silica has not been undertaken yet. The structure effects on reaction activity are often observed in the particle size within the $1-3 \mathrm{~nm}$ range, where the fraction of surface sites with different coordination number are expected to vary dramatically. ${ }^{22}$ Because of the complexity of catalysts as well as the sensitivity of Pt catalysts to carbon contaminations, it is difficult to establish a reliable correlation between the observed activity and the Pt particle size. In this paper, we report our study of controlling Pt particle size prepared by SOMC/M (refilling) under elevated pressure conditions to avoid carbon contamination of the surface.

\section{EXPERIMENTAL SECTION}

2.1. Catalysts Preparation. The starting $\mathrm{Pt} / \mathrm{SiO}_{2}$ catalyst was prepared using the classical ion exchange (IE) technique. ${ }^{23}$ The latter was subsequently used as a "host" material to synthesize different $\mathrm{Pt} / \mathrm{SiO}_{2}$ catalysts showing gradual increase in size using SOMC/M. The silica support used in the present work was a nonporous Aerosil 300 with a specific surface area of $280.15 \pm 1.57 \mathrm{~m}^{2} / \mathrm{g}$.

2.2. Preparation of Highly Dispersed "Host" Material $\mathrm{Pt} / \mathrm{SiO}_{2}$ Catalyst. The preparation of the monometallic silicasupported platinum was achieved by the ion-exchange method following the procedure already described ${ }^{17,24}$ using tetramine platinum hydroxide $\left[\mathrm{Pt}\left(\mathrm{NH}_{3}\right)_{4}\right](\mathrm{OH})_{2}$. Prior to metal salt addition, about $20 \mathrm{~g}$ of the silica support was treated under flowing dry air at $500{ }^{\circ} \mathrm{C}$ for $6 \mathrm{~h}$. The pretreated silica was contacted with $300 \mathrm{~mL}$ of deionized water and stirred during $30 \mathrm{~min}$. The $\mathrm{pH}$ of the solution was adjusted from 9.5 to 10 by adding $\mathrm{NH}_{4} \mathrm{OH}$, to facilitate ion exchange of the complex cations $\left[\mathrm{Pt}\left(\mathrm{NH}_{3}\right)_{4}\right]^{2+}$ with the silica surface (eq 1$)$. The suspension was stirred during $3 \mathrm{~h}^{25}$ Then, the solution of the platinum precursor $\left[\mathrm{Pt}\left(\mathrm{NH}_{3}\right)_{4}(\mathrm{OH})_{2}\right]\left(\mathrm{C}=0.01 \mathrm{~mol} \mathrm{~L}^{-1}\right)$ was added dropwise to the silica suspension, in order to prepare $\mathrm{Pt} / \mathrm{SiO}_{2}$ catalyst (eq 2). The mixture was stirred for $24 \mathrm{~h}$ at RT and filtered. The sample was washed with water until the $\mathrm{pH}$ becomes neutral and further dried under air for $18 \mathrm{~h}$ at $80^{\circ} \mathrm{C}$. The solid obtained was then reduced under flowing hydrogen at $300{ }^{\circ} \mathrm{C}$ for $3 \mathrm{~h}$ and stored at room temperature in air. ${ }^{26}$

$$
\begin{aligned}
& \equiv \mathrm{Si}-\mathrm{OH}+\mathrm{NH}_{4} \mathrm{OH} \rightarrow\left[\equiv \mathrm{SiO}^{-}\right]_{2}\left[\mathrm{NH}_{4}{ }^{2+}\right]+\mathrm{H}_{2} \mathrm{O} \\
& 2\left[\equiv \mathrm{SiO}^{-}\right]_{2}\left[\mathrm{NH}^{4+}\right]+\mathrm{Pt}\left(\mathrm{NH}_{3}\right)_{4}(\mathrm{OH})_{2} \\
& \left.\quad \rightarrow\left[\mathrm{SiO}_{2}^{-}\right]_{2} \mathrm{Pt}\left(\mathrm{NH}_{3}\right)_{4}{ }^{2+}\right]+2 \mathrm{NH}_{4} \mathrm{OH}
\end{aligned}
$$

2.3. Preparation of Monometallic Pt Supported on Silica Using the SOMC/M Approach. Before starting the $\mathrm{SOMC} / \mathrm{M}$, the "host" material was thoroughly characterized using several characterization techniques, such as chemical analysis, $\mathrm{H}_{2}$ chemisorption, and electron microscopy. The Pt loading of the host was $1.12 \mathrm{wt} \%$ as determined from ICP. In this manuscript, the host will be referred to as $\mathrm{Pt}(1.12) \mathrm{Si}_{(\mathrm{IE})}$. The hydrogen adsorption isotherm was measured by a static volumetric apparatus at $25{ }^{\circ} \mathrm{C} .{ }^{27,28}$ By assuming a stoichiometry of $2 \mathrm{H} /$ surface $\mathrm{Pt}^{29}$ the number of Pt surface atoms $\left(\mathrm{Pt}_{\mathrm{s}}\right)$ was $48.32 \mu \mathrm{mol}$. The resulting metal dispersion was ca. $87 \%$. Assuming a spherical shape of the particle, ${ }^{30}$ the particle size was estimated to be around $1.3 \mathrm{~nm}$. We have to keep in mind that particle with size as small as $1 \mathrm{~nm}$ or even below can be detected by our electron microscopic facilities. Supported monometallic Pt catalysts with different particle sizes were prepared by hydrogenolysis of platinum(II) acetylacetonate complex $\left(\mathrm{Pt}(\mathrm{acac})_{2}\right)$ on the $\mathrm{Pt}(1.12) \mathrm{Si}_{\text {(IE) }}$ monometallic starting material (eq 3 ), which was previously covered by irreversibly adsorbed hydrogen. Note that the amount of $\mathrm{Pt}$ introduced should correspond exactly to the amount of $\mathrm{Pt}_{\mathrm{s}}$ on the surface. ${ }^{29}$

$$
\mathrm{Pt}(\mathrm{acac})_{2}+2 \mathrm{Pt}_{\mathrm{s}}-\mathrm{H} \rightarrow 3 \mathrm{Pt}_{\mathrm{s}}+2(\mathrm{acac}) \mathrm{H}
$$

The reaction between $\mathrm{Pt}$ complex molecules and Pt hydride species occurred at $25{ }^{\circ} \mathrm{C}$ in dry and degassed toluene as solvent during $8 \mathrm{~h}$ under hydrogen atmosphere. The mixture was stirred overnight at RT and filtered. Then, the solid was washed many times with $200 \mathrm{~mL}$ of toluene and further dried under a flow of $\mathrm{H}_{2}$ for $18 \mathrm{~h}$ at $80{ }^{\circ} \mathrm{C}$. The obtained solid was finally reduced under flowing hydrogen at increasing temperature from 25 to $400{ }^{\circ} \mathrm{C}$, maintained at $400{ }^{\circ} \mathrm{C}$ for $3 \mathrm{~h}$, evacuated, and then stored at room temperature in air.

\section{CHARACTERIZATION TECHNIQUES}

3.1. Hydrogen Chemisorption Measurements. As explained above, hydrogen chemisorption was performed in a static volumetric apparatus, ${ }^{30}$ at $25{ }^{\circ} \mathrm{C}$ under increasing pressure. Before $\mathrm{H}_{2}$ adsorption, the sample was treated in situ at $350{ }^{\circ} \mathrm{C}$ under flowing hydrogen during $2 \mathrm{~h}$ to fully reduce the platinum and then evacuated under dynamic vacuum $\left(10^{-5}\right.$ mbar) at $350{ }^{\circ} \mathrm{C}$ during $2 \mathrm{~h}$ to completely remove adsorbed hydrogen (physisorbed and chemisorbed). The number of $\mathrm{Pt}_{\mathrm{s}}$ was determined by assuming a stoichiometry of $2 \mathrm{H} / \mathrm{Pt}_{\mathrm{s}}{ }^{25}{ }^{25}$ The total number of $\mathrm{Pt}$ atoms $(\mathrm{Pt})$ was measured by elemental analysis, and the resulting metal dispersion was $D=\mathrm{Pt}_{\mathrm{s}} / \mathrm{Pt}$. Assuming a spherical shape, the particle size was estimated from $D$, by eq 4

$$
D=6 M / \rho d N_{\mathrm{a}} S_{\mathrm{a}}
$$

where $M$ is the atomic weight; $\rho$ is the density of the metal $(\mathrm{Pt}$, $\left.\rho=21.45 \mathrm{~g} \mathrm{~cm}^{-3}\right)$; $N_{\mathrm{a}}$ is Avogadro's number; and $S_{\mathrm{a}}$ is the area of each surface Pt atom $\left(\mathrm{Sa}=8.0 \times 10^{-20} \mathrm{~m}^{2} /\right.$ atom $)$. 
3.2. UV-Vis Spectroscopy. The reaction of $\mathrm{Pt}(\mathrm{acac})_{2}$ with silica surface and toluene as a solvent was followed by UV-vis spectroscopy. Using the same conditions used for grafting $\mathrm{Pt}$ (see section 2.3), the amount of $\mathrm{Pt}(\mathrm{acac})_{2}$ in the liquid obtained after filtration of the silica suspension was determined from the UV-vis spectra recorded in transmission mode on a Thermoscientific evolution 600 spectrometer equipped with a $10 \mathrm{~mm}$ quartz cell. The concentration of $\mathrm{Pt}$ was determined from the intensity of the absorption peak at $350 \mathrm{~nm}$ (see the Supporting Information; Figure S1), which corresponds to the maximum absorbance coefficient of $\mathrm{Pt}(\mathrm{acac})_{2}$ in toluene, using the Beer-Lambert law.

3.3. CO Chemisorption Followed by FTIR. The CO adsorption experiments were performed using a PerkinElmer 1760 spectrometer, equipped with an MCT detector. The IR spectra were collected after 128 scans in transmission mode using a resolution of $4 \mathrm{~cm}^{-1}$. All spectra were recorded on thin self-supported wafers. ${ }^{31}$ The wafers were first reduced in $\mathrm{H}_{2}$ (1 bar, $300{ }^{\circ} \mathrm{C}, 2{ }^{\circ} \mathrm{C} / \mathrm{min}, 1 \mathrm{~h}$ ), followed by outgassing in vacuum $\left(P=10^{-3} \mathrm{mbar}\right)$ for $1 \mathrm{~h}$ at $300{ }^{\circ} \mathrm{C}$ to remove the adsorbed hydrogen. The wafers were later cooled to RT and exposed to 300 mbar of CO. Subsequently, the sample was evacuated for $15 \mathrm{~min}$ at $10^{-3} \mathrm{mbar}$ to remove weakly adsorbed $\mathrm{CO}$ molecules. The final FTIR spectra were obtained by subtraction of the sample spectrum before and after $\mathrm{CO}$ chemisorption. ${ }^{32}$ To measure the accessible area of the $\mathrm{Pt}$ particle by using different probe molecules, we used a $\mathrm{CO}$ pulse chemisorption system equipped with a $\mathrm{CO}$ chemisorption device. This was carried out at RT by a pulse injection method. In principle, the sample was first purged with $\mathrm{He}$ for 1 $h$, and then a mixture of $\mathrm{H}_{2} / \mathrm{He}(10 \%)$ was introduced onto the catalysts, which were reduced at $350{ }^{\circ} \mathrm{C}$. $\mathrm{CO}$ pulses $(15$ $\mathrm{cm}^{3}$ ) were injected into the carrier gas intermittently after having cooled to RT, and the gaseous sample was detected by a TCD. By calculating Pt specific surface area from CO chemisorption data, it was assumed that $\mathrm{CO}$ was mostly chemisorbed in a linear form (IR spectroscopy) over Pt atoms $\left(\mathrm{CO} / \mathrm{Pt}_{\mathrm{s}}=1\right)$.

3.4. Electron Microscopy. High-angle annular dark fieldscanning transmission electron microscopy-electron energy loss spectroscopy (HAADF-STEM-EELS) was carried out with a Titan Cs-Probe Corrected (FEI Co.) microscope operated at $300 \mathrm{kV}$, equipped with a GIF Quantum from Gatan Inc. (Pleasanton, CA). Specimens were prepared by mixing the samples in pure ethanol solvent, and then a small amount of the resulting solution was placed onto copper $(\mathrm{Cu})$ grids having a holey-C layer of thickness of about $20 \mathrm{~nm}$. We used the HAADF-STEM technique to measure the particle size distribution as well as their crystallinity. Using the EELS technique, Pt-M4,5 (2122 eV), energy-loss edges were utilized to get Pt elemental maps, respectively.

3.5. DFT Calculations. For structural simulations of monometallic Pt nanoparticles, we have considered the three commonly FCC-derived truncated octahedral or cuboctahedral shapes characterized by the two main (100) and (111) facets and icosahedral shape characterized only by the (111) facet. We have started from the 38 -atom geometrical model of platinum $\left(\mathrm{Pt}_{38}\right)$ nanoparticle (size around $0.8 \mathrm{~nm}$ ) as the first magic number with the truncated octahedral shape and from the 13-atom geometrical model of the platinum $\left(\mathrm{Pt}_{13}\right)$ nanoparticle (size around $0.5 \mathrm{~nm}$ ) as the first magic number with both the cuboctahedral and icosahedral shapes. Atomic structures of truncated octahedron $\mathrm{Pt}_{n}$ nanoparticles $(n=116$,
260 , and 490 atoms) with larger sizes ranging from 1.4 up to $2.6 \mathrm{~nm}$ and cuboctahedron or icosahedron $\mathrm{Pt}_{n}$ nanoparticles $(n$ $=55,147,309$ atoms) with larger sizes ranging from 1.0 to 2.2 $\mathrm{nm}$, were built by a successive addition of one complete atomic layer in order to exactly determine upon optimization the size increase or the atomic layer thickness after each refilling run and compare with our experimental observations.

All calculations were carried out using the VASP simulation program $^{33-36}$ by employing the Perdew-Burke-Ernzerhof (PBE) exchange-correlation functional ${ }^{37}$ and the projectoraugmented plane wave (PAW) approach ${ }^{38}$ for electronelectron and electron-ion interaction descriptions, respectively. The valence electron configuration of $\mathrm{Pt}$ used in the PAW potential is $5 \mathrm{~d}^{9} 6 \mathrm{~s}^{1}$. The convergence criterion for the electronic self-consistent-field (SCF) cycles was set at $10^{-5} \mathrm{eV}$. For each nanoparticle, a full structural relaxation of the atomic coordinates was performed until the three components of the Hellmann-Feynman forces on each atom were below $0.01 \mathrm{eV} /$ Å.

3.6. Isobutane Catalytic Hydrogenolysis and Isomerization. All catalytic tests were conducted at atmospheric pressure in the gas phase using a fixed-bed isothermal flow reactor purchased from Process Integral Development and Engineering Technology (PI\&D Eng. \& Tech.). In a typical experiment, $100 \mathrm{mg}$ of the as-prepared catalyst (mesh size $250-500 \mu \mathrm{m}$ ) was loaded into a stainless-steel (SS-316) reactor (length $30 \mathrm{~cm}$ and $9 \mathrm{~mm}$ i.d.). The SS tube was equipped with a $20 \mu \mathrm{m}$ mesh size porous plate made from Hastelloy C, which was used to hold the catalyst inside the reactor. A type $\mathrm{K}$ thermocouple located within the catalyst bed was used to probe the temperature of the reaction. Calibrated Bronkhorst mass flow controllers (MFCs) were used to control the volumetric flow of all gases and the composition of the feed. A gas mixture with relative molar fraction of 1:10:9 of isobutane, $\mathrm{H}_{2}$, and $\mathrm{He}$, respectively, was used as a feed. Prior to the catalytic test, all samples were activated in situ under 10 $\mathrm{mL} / \mathrm{min} @ \mathrm{NTP} \mathrm{H}_{2}$ flow for $1 \mathrm{~h}$ at $300{ }^{\circ} \mathrm{C}\left(2{ }^{\circ} \mathrm{C} / \mathrm{min}\right)$, and then the catalyst was flushed with $\mathrm{He}$ for $30 \mathrm{~min}$. Finally, the catalyst temperature was adjusted to the required value, and the feed was then introduced.

The total volumetric flow was adjusted between 40 and 80 $\mathrm{mL} / \mathrm{min} @ \mathrm{NTP}$ in order to keep the conversion below 5\% under the present operating conditions of temperature and pressure. Online gas analysis of the products was performed on a Varian 450 GC equipped with an Agilent HP-Al/S capillary column. The main products of reaction detected at the reactor outlet are methane, propane, and $n$-butane. Traces of ethane and isobutylene were also sometimes detected. All catalytic data reported hereafter were obtained under steady state conditions. Blank tests conducted using silica showed no significant conversion under the present operating conditions. Repeatability of the catalytic tests was within $5 \%$. Carbon mass balance was always above $95 \%$. Turnover rates (TORs) were estimated by dividing the rate of the reaction (moles consumed of isobutane $/ \mathrm{mol}$ of metal/unit time) by surface metal atoms (metal dispersion values were obtained from the $\mathrm{H}_{2}$ chemisorption experiments).

\section{RESULTS AND DISCUSSION}

4.1. Hydrogenolysis of $\mathrm{Pt}(\mathrm{acac})_{2}$ on a Silica Blank Surface Followed by UV-Vis Spectroscopy. Before the addition of $\mathrm{Pt}(\mathrm{acac})_{2}$, silica (Aerosil from Degussa) was first treated under flowing $\mathrm{H}_{2}$ at $300{ }^{\circ} \mathrm{C}$ and then stored under $\mathrm{H}_{2}$ 
atmosphere at room temperature. The required amount of $\mathrm{Pt}(\mathrm{acac})_{2}$ degassed was carefully introduced into the reactor via a septum to minimize contact with air. The reaction of $\mathrm{Pt}(\mathrm{acac})_{2}$ with $\mathrm{SiO}_{2}$ was investigated at the following temperatures: 25, 50, and $100{ }^{\circ} \mathrm{C}$ (see the Supporting Information; Table S1). At the end of the reaction, the solid was filtered and washed with $200 \mathrm{~mL}$ of toluene. Then, the sample was dried in an oven, and the amount of grafted Pt was determined by elemental analysis. The amount of Pt in the liquid phase after filtration was measured by UV-vis spectroscopy.

The UV-vis spectra of $\mathrm{Pt}(\mathrm{acac})_{2}$ dissolved in toluene exhibits a maximum absorption at $\lambda_{\max }=350 \mathrm{~nm}$, which is characteristic of the acetylacetonate chromophore. Table S1 (in the Supporting Information) summarizes the results of $\mathrm{Pt}(\mathrm{acac})_{2}$ reaction with silica at different temperatures. We can clearly see that the amounts of Pt as determined by ICP and UV-vis are in good agreement. Moreover, the yield of the reaction of silica with $\mathrm{Pt}(\mathrm{acac})_{2}$ increases with increasing the temperature, as judged by the increasing Pt content of the silica substrate. Nevertheless, the amount of Pt grafted on silica was found to be very small (less than $0.1 \%$ ) and can be neglected at temperatures up to $25^{\circ} \mathrm{C}$. Accordingly, all grafting reactions of refilling were performed at $25^{\circ} \mathrm{C}$, and all obtained solids were thoroughly washed, prior to reduction, in order to minimize the amount of $\mathrm{Pt}(\mathrm{acac})_{2}$ that could interact weakly with the silica surface.

4.2. Hydrogenolysis of $\mathrm{Pt}(\mathrm{acac})_{2}$ on Supported Platinum (Refilling Reaction). Refilling reaction was carried out using Pt $(1.12 \%)_{(\mathrm{IE})}$ as a starting material, and elemental analysis gives $1.12 \% \mathrm{Pt}$ loading. From hydrogen chemisorption, the dispersion of the sample is 0.87 suggesting particles size of about $1 \mathrm{~nm}$. TEM analysis for the starting material (0G) shows an average particle size of $1.1 \mathrm{~nm}$. After reduction at $350{ }^{\circ} \mathrm{C}$ under flow of pure hydrogen, the catalyst was transferred to a solution of $\mathrm{Pt}(\mathrm{acac})_{2}$ in toluene. As expected, the overall $\mathrm{Pt}$ metal loading steadily increases with successive refilling. It should be noted that the ligand present on the precursor (acac) is eliminated very quickly as acacH by reaction with $\mathrm{Pt}-$ $\mathrm{H}$, and this is a general phenomenon in SOMC/M. ${ }^{39}$ Therefore, the ligand is not perturbing the approach of the new $\operatorname{Pt}(\mathrm{acac})_{2}$ to the surface.

$\mathrm{H}_{2}$ Chemisorption. Indeed, after two refilling stages, the metal loading increased from 1.12 to $1.74 \mathrm{wt} \% \mathrm{Pt}$. The amount of platinum $\left(\mathrm{Pt}_{\mathrm{s}}{ }^{i}\right)$ present on the surface of the catalyst after each refilling stage $i$ was assessed using $\mathrm{H}_{2}$ chemisorption. The $\mathrm{H}_{2}$ chemisorption isotherms of all prepared $\mathrm{Pt} / \mathrm{SiO}_{2}$ catalysts are depicted in Figure S2 (see the Supporting Information for more details). Clearly, the total amount of $\mathrm{H}_{2}$ chemisorbed related to the total amount of $\mathrm{Pt}(\mathrm{H} / \mathrm{Pt})$ decreases after each refilling stage. This is particularly true after two refilling steps in two stages, which would indicate a growth of the Pt-supported particles. The amounts of $\mathrm{Pt}_{\mathrm{s}}{ }^{i}$ $(\mu \mathrm{mol} / \mathrm{g})$ obtained from $\mathrm{H}_{2}$ chemisorption measurements are summarized in Table S2 (in the Supporting Information). As a matter of fact, the coverage of the platinum surface by platinum atoms evaluated as $\Theta=\mathrm{Pt}_{\mathrm{s}}{ }^{i+1} / \mathrm{Pt}_{\mathrm{s}}{ }_{\mathrm{s}}$ is always close to 1 , which indicates a selective deposition of $\mathrm{Pt}$ on the metallic surface with no significant deposition on the silica surface using the current SOMC protocol.

TEM Analysis. Despite the low amounts of platinum added to the catalyst, the dispersion drops considerably after each refilling run, indicating an effective growth of the particles. The particle size distributions of the so-called "host" sample stating 1.12 wt \% are shown in Figure 1 and Table 1. The average size
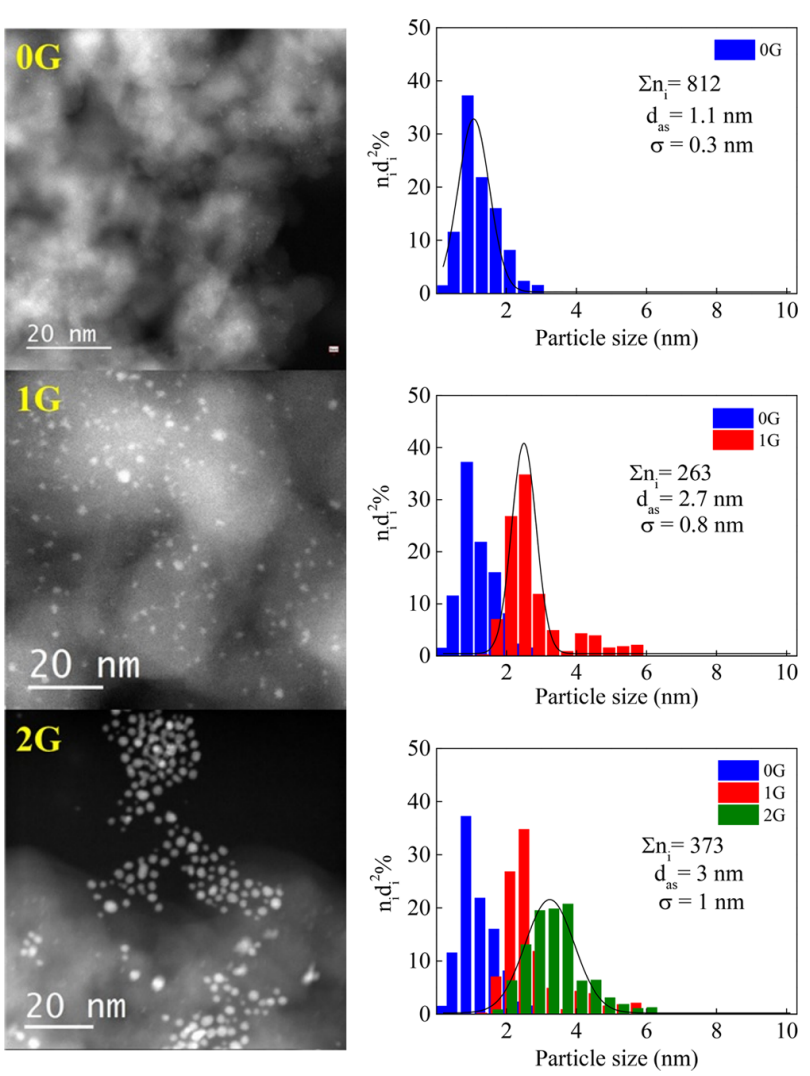

Figure 1. STEM images and histogram of particle size distributions of $\mathrm{Pt} / \mathrm{SiO}_{2}$ for different generations (0G, $1 \mathrm{G}$, and $2 \mathrm{G}$ ).

of particles is around $1.1 \pm 0.3 \mathrm{~nm}$. After the first and second refilling runs, the average particle size is shifted toward $2.3 \pm$ $0.8 \mathrm{~nm}$ and $3 \pm 1 \mathrm{~nm}$, respectively. Again, the size distribution has slightly broadened the particle range between 1.7 and 6 $\mathrm{nm}$. It should be noted that the particle size is becoming broader and broader after each refilling. There are several possibilities that could be envisaged for such a phenomenon. We prefer to say that very likely that if the stoichiometry of $\mathrm{Pt}(\mathrm{acac})_{2} /$ initial $\mathrm{Pt}-\mathrm{H}$ is not perfectly respected then there is some $\mathrm{Pt}(\mathrm{acac})_{2}$ which is deposited directly on the silica surface, leading to a broadening of particle size distribution. This phenomenon is obviously linked to the number of refillings.

CO Chemisorption. The results of $\mathrm{Pt}$ dispersion and average particle size as estimated using $\mathrm{CO}$ pulse chemisorption and TEM data are summarized in Table 1 . As pointed out above, and in agreement with $\mathrm{H}_{2}$ chemisorption measurements, the dispersion decreases after each refilling stage. In general, there is a good agreement between the values of dispersion obtained by TEM and $\mathrm{H}_{2}$. However, the values obtained from $\mathrm{CO}$ chemisorption differ quite significantly from the two other techniques because it has been shown that the stoichiometry of $\mathrm{CO}$ chemisorbed on platinum depends quite significantly on particle size. Therefore, the results of particle size and dispersion deduced from $\mathrm{CO}$ chemisorption have not been considered as reliable enough in this paper 
Table 1. Pt/SiO 2 at Different Refilling Run ${ }^{a}$

\begin{tabular}{|c|c|c|c|c|c|c|c|c|}
\hline \multirow[b]{2}{*}{ catalyst code } & \multirow[b]{2}{*}{ Pt wt \% } & \multicolumn{2}{|c|}{ TEM } & \multicolumn{2}{|c|}{$\mathrm{H}_{2}$ chemisorption } & \multicolumn{2}{|c|}{$\mathrm{CO}$ chemisorption } & \multirow{2}{*}{$\frac{\text { theoretical particle size expected }}{(\mathrm{nm})}$} \\
\hline & & $d_{\mathrm{as}}(\mathrm{nm})$ & $D \% * *$ & $d_{\mathrm{as}}^{*}(\mathrm{~nm})$ & $D \%$ & $d_{\mathrm{as}}(\mathrm{nm})$ & $D \% * *$ & \\
\hline OG & 1.12 & $1.1 \pm 0.3$ & 82 & 1.3 & 87 & 1.8 & 46 & - \\
\hline $1 G$ & 1.55 & $2.3 \pm 0.8$ & 42 & 2.2 & 51 & 3.12 & 36 & 1.9 \\
\hline $2 \mathrm{G}$ & 1.74 & $3 \pm 1$ & 38 & 2.9 & 39 & 3.5 & 35 & 2.7 \\
\hline
\end{tabular}

${ }^{a} d_{\mathrm{as}}$ : average particle size in surface, $d_{\mathrm{as}}=\Sigma n_{i} d_{i}^{3} / \Sigma n_{i} d_{i}^{2} ; D \% * *$ : dispersion calculated by specific surface area of Pt $\left(S_{\mathrm{sp}}\right)$ corresponding to $d_{\mathrm{as}} ; d_{\mathrm{as}}^{*}$ : calculated particle size from $D$ measured by $\mathrm{H}_{2}$ chemisorption and assuming a spherical shape.

4.3. Shape of Pt Particle. The morphology of supported $\mathrm{Pt}$ nanoparticles often depends on the nature of the Pt precursor, the preparation method, and the thermal treatment. Hereafter, the shape of the Pt nanoparticle after successive refilling runs was studied by high-resolution transmission electron microscopy (HR-TEM). The $1 \mathrm{G} \mathrm{Pt} / \mathrm{SiO}_{2}$ catalyst seems to exhibit a spherical-like shape similar to the one observed in the case of the "staring material". After the second refilling (2G), it can be seen from HR-TEM that the Pt nanoparticles with an average diameter around $2 \mathrm{~nm}$ are truncated cuboctahedra (see inset in Figure 2). After the

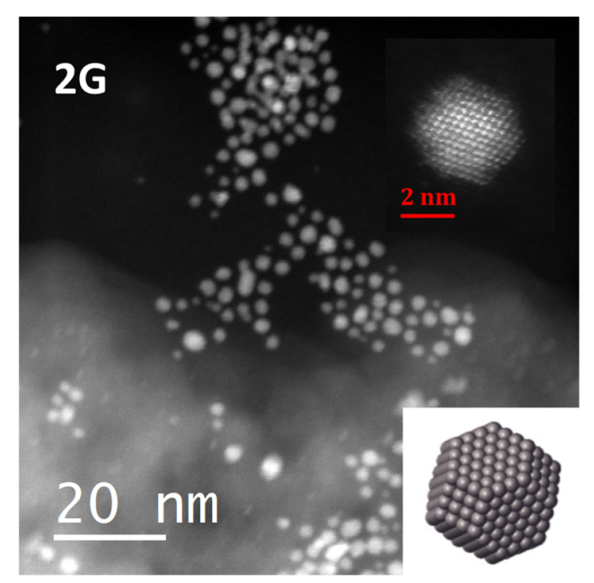

Figure 2. Pt particle shape after second refilling.

second refilling (2G), truncated cuboctahedral particles are observed. It is noteworthy that, although cuboctahedral Pt particles could not be detected in $\mathrm{Pt} / \mathrm{SiO}_{2}$ (0G and $1 \mathrm{G}$ ), their presence cannot be completely ruled out. Moreover, previous reports claimed that $\mathrm{Pt}$ particles could change their cuboctahedral shape into a spherical one when treated under reducing or oxidizing atmosphere. ${ }^{40,41}$

4.4. CO Chemisorption Followed by FTIR. The IR spectrum found upon chemisorption of $\mathrm{CO}$ on a metallic surface can be affected by the size and morphology of the particle. IR spectra of adsorbed $\mathrm{CO}$ on $\mathrm{Pt} / \mathrm{Al}_{2} \mathrm{O}_{3}$ give two $\nu_{\mathrm{CO}}$ bands: the first one between 2090 and $2040 \mathrm{~cm}^{-1}$ is attributed to linearly adsorbed $\mathrm{CO}$ on $\mathrm{Pt}$ atom, while the second one around $1860-1780 \mathrm{~cm}^{-1}$ is attributed to CO bridging two or more $\mathrm{Pt}$ atoms. ${ }^{42}$ The intensity of the bridging $\mathrm{CO}$ band is very weak, and this type of mode is difficult to be detected in the case of Pt.

The metal-CO interaction is often described using the model proposed by Blyholder. ${ }^{43,44}$ To simplify a rather controversial field, Blyholder explained the higher strength of bonds between the metal and $\mathrm{CO}$ in terms of the formation of a $\pi$ bond due to partial filling of $\mathrm{d}$ orbitals toward the $\pi^{*}$ of
CO. This leads to a weakening of the carbon to oxygen bond and hence a shift in the IR stretching frequency of $\mathrm{CO}$ with respect to the gaseous state. However, another parameter was found to take place for $\mathrm{CO}$ adsorbed on platinum. The coupling could occur if the $\mathrm{CO}$ molecules are close enough from each other, which complicates considerably the interpretation of the IR spectra. Two consequences can be derived from this model as follows:

(i) Increasing the particle size change of the coordination number of a surface metal atom, which leads to a possible change in the adsorption site for CO. The increased competition of $\mathrm{CO}$ and the other neighboring atoms decreases the back bonding from the metal adsorption site to the $\pi^{*}$ orbitals of $\mathrm{CO}$, leading to an increased frequency. Indeed, numerous studies of $\mathrm{CO}$ adsorption have attempted to correlate the $\mathrm{CO}$ frequency with the coordination of the $\mathrm{Pt}$ atoms at the surface and observed an increase in the $\nu(\mathrm{CO})$ stretching frequency with increasing particle size. ${ }^{45}$ Moreover, other investigators reported a linear relationship between the $\nu(\mathrm{CO})$ stretching frequency and the $\mathrm{Pt}$ coordination number. ${ }^{46,47}$ The authors claimed that the metallic atoms surrounding the adsorbing center would compete for electrons with the adsorbed $\mathrm{CO}$ molecule. Accordingly, one would expect a change in the competition for electrons between $\mathrm{CO}$ and neighboring metal atoms depending on the location of the metal center. In fact, the coordination number would vary between different facets, edges, and corners. Therefore, M$\mathrm{CO}$ bond strength could be significantly affected by a change in the coordination number of the surface metal and, hence, results in a change in the position of the $\nu(\mathrm{CO})$ frequency.

(ii) $\mathrm{CO}$ coverage-dependent frequency shift would be caused by a change in the number of competing $\mathrm{CO} 2 \pi^{*}$ orbitals for the available $d$ electrons of the metal, though the $\mathrm{CO}$ coverage is significantly reflected also by dipole-dipole coupling between chemisorbed species. ${ }^{48-52}$

Facing such difficulty of interpretation, we would like to give the results obtained on $0 \mathrm{G}, 1 \mathrm{G}$, and $2 \mathrm{G}$ under identical conditions (Figure 3). By increasing the particle size the $\nu(\mathrm{CO})$ frequency increases from 2086 to $2095 \mathrm{~cm}^{-1}$. This increase of frequency is too small to reflect only one parameter. We will see in the DFT part that when we increase the size from 1 to $3 \mathrm{~nm}$ the number of exposed platinum atoms on faces increases quite a lot. However, simultaneously the number of atoms in the edges increases also quite significantly. These two parameters cannot be unambiguously separated. We just prefer to say that the overall back bonding seems to decrease when the particle size increases.

4.5. DFT Calculations. The atomic configurations for truncated octahedron $\mathrm{Pt}_{n}$ nanoparticles $(n=38,116,260$, and 490 atoms), cuboctahedron, and icosahedron $\mathrm{Pt}_{n}$ nanoparticles ( $n=55,147$, and 309 atoms), as considered by DFT, are illustrated in Figure 4. The obtained particle size (in $\mathrm{nm}$ ) for 


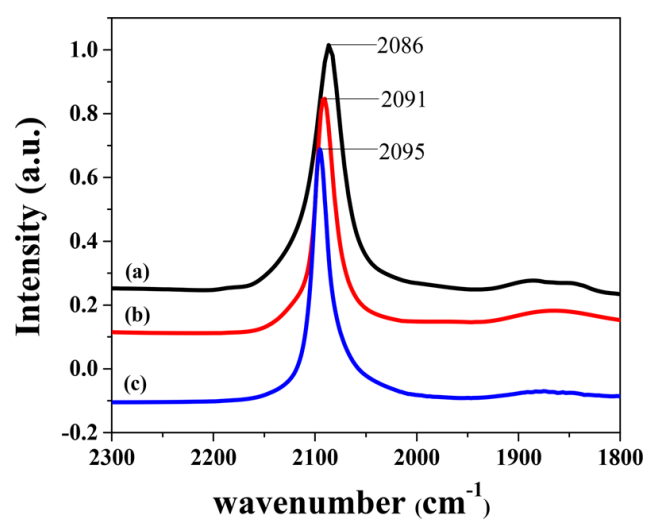

Figure 3. FTIR spectra of $\mathrm{CO}$ on $\mathrm{Pt} / \mathrm{SiO}_{2}$ (a) starting material (0G), (b) first refilling (1G), and (c) second refilling (2G).

each nanoparticle after a full structural optimization is given above each structure.

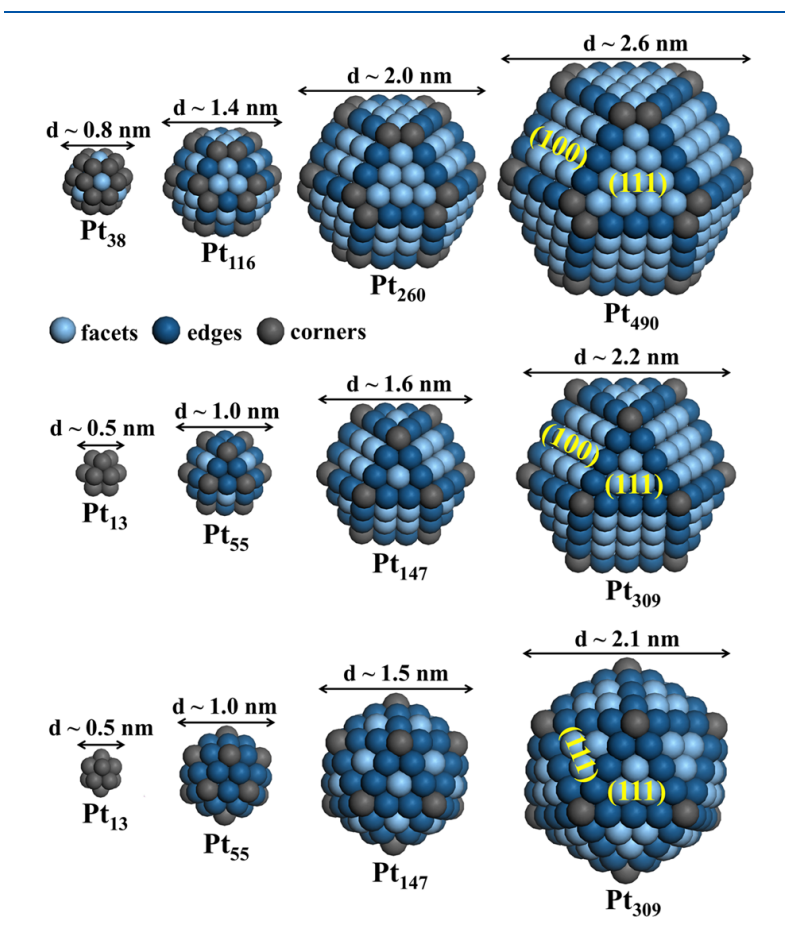

Figure 4. DFT-based optimized atomic configurations for truncated octahedron $\mathrm{Pt}_{n}$ nanoparticles $(n=38,116,260$, and 490 atoms; located on the top) with different sizes ranging from $0.8 \mathrm{~nm}$ (for 38 atoms) up to $2.6 \mathrm{~nm}$ (for 490 atoms), for cuboctahedron $\mathrm{Pt}_{n}$ nanoparticles $(n=13,55,147$, and 309 atoms; located in the middle) with different sizes ranging from $0.5 \mathrm{~nm}$ (for 13 atoms) up to $2.2 \mathrm{~nm}$ (for 309 atoms), and for icosahedron $\mathrm{Pt}_{n}$ nanoparticles $(n=$ $13,55,147$, and 309 atoms; located on the bottom) with different sizes ranging from $0.5 \mathrm{~nm}$ (for 13 atoms) up to $2.1 \mathrm{~nm}$ (for 309 atoms).

For truncated octahedron $\mathrm{Pt}_{38}$, the obtained size upon optimization is around $0.8 \mathrm{~nm}$. Adding one complete atomic layer led to the formation of $\mathrm{Pt}_{116}$ with an optimized larger size around $1.4 \mathrm{~nm}$. Further additions of one and two closed atomic layers led to the formation of $\mathrm{Pt}_{260}$ and $\mathrm{Pt}_{490}$ with larger sizes around 2.0 and $2.6 \mathrm{~nm}$ after full geometrical optimizations, respectively. For cuboctahedron or icosahedron
$\mathrm{Pt}_{13}$, the obtained optimized size is $0.5 \mathrm{~nm}$, and the addition of one complete atomic layer led to the formation of $\mathrm{Pt}_{55}$ with a larger size around $1.0 \mathrm{~nm}$. Adding one and two more complete atomic layers led to the formation of $\mathrm{Pt}_{147}$ and $\mathrm{Pt}_{309}$ with larger sizes around 1.6/1.5 and 2.2/2.1 nm, respectively. As a conclusion, DFT calculations showed a Pt particle size increase of around $0.6 \mathrm{~nm}$ after each refilling run.

Table 2 summarizes the total number of $\mathrm{Pt}$ atoms located on corners, edges, and facets in the atomic structures of truncated octahedron, cuboctahedron, and icosahedron $\mathrm{Pt}_{n}$ nanoparticles presented in Figure 4. Overall, the number of atoms in both the edges and facets increases with increasing particle size using the three types of particle shape. However, the number of edge atoms separating $\mathrm{Pt}(111)$ or/and $\mathrm{Pt}(100)$ facets is increasing more rapidly in the case of icosahedron than that of cuboctahedron than that of the truncated octahedron, while the number of atoms located on $\mathrm{Pt}(100)$ and/or $\mathrm{Pt}(111)$ facets is increasing more rapidly in the cases of cuboctahedron and truncated octahedron than that of icosahedron. The impact of surface structure on the catalytic results is discussed in the next subsection.

4.6. Platinum Particle Size Effects on Isobutane Hydrogenolysis and Isomerization. As explained just above in subsection 4.5, the DFT calculations of the atomic configurations for truncated octahedron, cuboctahedron, and icosahedron $\mathrm{Pt}_{n}$ nanoparticles within the $0.5-2.6 \mathrm{~nm}$ size range clearly showed that the ratio between the total number of $\mathrm{Pt}$ atoms located on edges versus corners varies from 0 to 7.5 , and the ratio between the total number of $\mathrm{Pt}$ atoms located on facets versus corners varies from 0 to 6.5. As seen in Figure 1, the large majority of the particles in the starting material $(0 \mathrm{G})$ is about $1 \mathrm{~nm}$, and then this value goes to $2.2-$ $2.4 \mathrm{~nm}$ in $1 \mathrm{G}$ and $3.0-4.0 \mathrm{~nm}$ for $2 \mathrm{G}$. As a consequence, we are clearly in the suitable range to observe the change in selectivity and activity, related to the change in the edges/ corners and facets/corners atom number ratios.

$\mathrm{Pt} / \mathrm{SiO}_{2} \mathrm{NP}$ samples prepared by SOMC were tested in the catalytic hydrogenolysis and isomerization of isobutane. The conversion was directly linked to contact time, as shown in Figure 5, by using three different flows: 40,60 , and $60 \mathrm{~mL}$ $\mathrm{min}^{-1}$ at $300^{\circ} \mathrm{C}$ for a constant contact time. Therefore, we can assume that the reactions were conducted under kinetic control as suggested by G. J. K. Acres, ${ }^{53}$ which is a general assumption in heterogeneous catalysis in dynamic differential reactors. Under this condition, the products formed were methane, propane, $n$-butane, and isobutylene, as shown in Table S3 (see the Supporting Information).

The catalytic activities and selectivities of Pt monometallic catalysts prepared via SOMC are summarized in Figures S3 and S4 (in the Supporting Information). The isobutane TORs were calculated using the number of exposed atoms on surface deduced from the $\mathrm{H}_{2}$ chemisorption measurements. As shown in Figure $6 \mathrm{a}$, the catalytic activity of isobutane global conversion TOR decreases with increasing $\mathrm{Pt}$ particle size (black square on Figure 6a). The isobutane TOR over the Pt NP $1.3 \mathrm{~nm}$ catalyst is $3.5 \times 10^{-3} \mathrm{~s}^{-1}$, which is about 1.7 times higher than that on the Pt NP $3.4 \mathrm{~nm}$ catalyst $\left(2.2 \times 10^{-3} \mathrm{~s}^{-1}\right)$. In contrast, the activity for hydrogenolysis $\left(\mathrm{TOR}_{\mathrm{H}}\right)$ dramatically decreases with increasing Pt particle size, whereas the activity for isomerization $\left(\mathrm{TOR}_{\mathrm{I}}\right)$ increases until $\mathrm{Pt}$ particle size around $2.3 \mathrm{~nm}$ and then decreases for larger particle. The selectivity for hydrogenolysis observed on Figure $6 \mathrm{~b}$ over a series of Pt NPs decreases with increasing particle size up to 
Table 2. Number of Pt Atoms Located on Corners $\left(N_{\text {corners }}\right)$, Edges $\left(N_{\text {edges }}\right)$, and Facets $\left(N_{\text {facets }}\right)$ in the Atomic Structures of Truncated Octahedron, Cuboctahedron, and Icosahedron $\mathrm{Pt}_{n}$ Nanoparticles Reported in Figure 4

\begin{tabular}{|c|c|c|c|c|c|c|c|}
\hline & & size $(\mathrm{nm})$ & $N_{\text {corners }}$ & $N_{\text {edges }}$ & $N_{\text {facets }}$ & $N_{\text {edges }} / N_{\text {corners }}$ & $N_{\text {facets }} / N_{\text {corners }}$ \\
\hline \multirow[t]{4}{*}{ truncated octahedron } & $\mathrm{Pt}_{38}$ & 0.8 & 24 & 0 & 8 & 0 & 0.3 \\
\hline & $\mathrm{Pt}_{116}$ & 1.4 & 24 & 24 & 30 & 1.0 & 1.2 \\
\hline & $\mathrm{Pt}_{260}$ & 2.0 & 24 & 48 & 72 & 2.0 & 3.0 \\
\hline & $\mathrm{Pt}_{490}$ & 2.6 & 24 & 72 & 134 & 3.0 & 5.6 \\
\hline \multirow[t]{4}{*}{ cuboctahedron } & $\mathrm{Pt}_{13}$ & 0.5 & 12 & 0 & 0 & 0 & 0 \\
\hline & $\mathrm{Pt}_{55}$ & 1.1 & 12 & 24 & 6 & 2.0 & 0.5 \\
\hline & $\mathrm{Pt}_{147}$ & 1.6 & 12 & 48 & 32 & 4.0 & 2.6 \\
\hline & $\mathrm{Pt}_{309}$ & 2.2 & 12 & 72 & 78 & 6.0 & 6.5 \\
\hline \multirow[t]{4}{*}{ icosahedron } & $\mathrm{Pt}_{13}$ & 0.5 & 12 & 0 & 0 & 0 & 0 \\
\hline & $\mathrm{Pt}_{55}$ & 1.0 & 12 & 30 & 0 & 2.5 & 0 \\
\hline & $\mathrm{Pt}_{147}$ & 1.5 & 12 & 60 & 20 & 5.0 & 1.6 \\
\hline & $\mathrm{Pt}_{309}$ & 2.1 & 12 & 90 & 60 & 7.5 & 5.0 \\
\hline
\end{tabular}

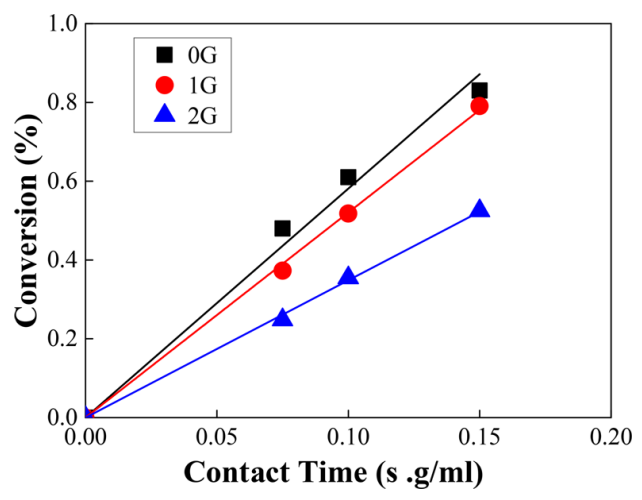

Figure 5. Influence of contact time on conversion of isobutane over $\mathrm{Pt} / \mathrm{SiO}_{2}$ prepared by SOMC (flow $=40,60$, and $80 \mathrm{~mL} / \mathrm{min}$, $\mathrm{H}_{2}:$ isoC $_{4}=10: 1$, mass of catalyst $=100 \mathrm{mg}$ ) at $300{ }^{\circ} \mathrm{C}$.

around $2 \mathrm{~nm}$ and then stabilizes up to $3.4 \mathrm{~nm}$ Pt particle size. For isomerization, the selectivity increased with increasing the particle size to $2 \mathrm{~nm}$ and then decreased again upon a further Pt particle size to $3 \mathrm{~nm}$.

To summarize, the global surface activity (TOR of isobutane) decreases with increasing the $\mathrm{Pt} / \mathrm{SiO}_{2}$ particle size. When trying to ascribe a given reaction to a given geometry, $\mathrm{TOR}_{\mathrm{H}}$ (hydrogenolysis) and $\mathrm{TOR}_{\mathrm{I}}$ (isomerization) varied in the opposite direction; $\mathrm{TOR}_{\mathrm{H}}$ dramatically decreases; while $\mathrm{TOR}_{\mathrm{I}}$ slightly increased with increasing the particles size. Let us recall here that $\mathrm{H}_{2}$ chemisorption and DFT calculations showed that the surface of small Pt nanoparticles $(1.3 \mathrm{~nm})$ is mainly composed of low coordination $\mathrm{Pt}$ atoms, while larger ones of $3.4 \mathrm{~nm}$ exhibit more facets than corners and edges. It is therefore suggested that hydrogenolysis might preferentially occur on low coordination surface platinum atoms (corners and edges), while isomerization occurs mostly on the facets.

\section{CONCLUSIONS}

The interaction between the $\mathrm{Pt}(\mathrm{acac})_{2}$ and silica-supported platinum surfaces was carried out to define the experimental conditions allowing the controlled variation of the particle size of $\mathrm{Pt} / \mathrm{SiO}_{2}$ catalysts by $\mathrm{SOMC} / \mathrm{M}$ refilling protocol. In this study, we found the experimental conditions where there was no reaction between $\mathrm{Pt}(\mathrm{acac})_{2}$ and silica surface, while hydrogenolysis of the platinum(II) acetylacetonate complex

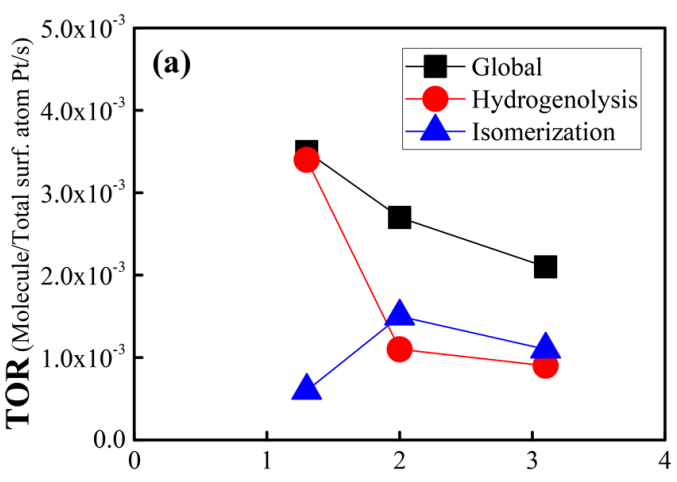

Pt particle size by $\mathrm{H}_{2} \mathrm{Chem}(\mathrm{nm})$

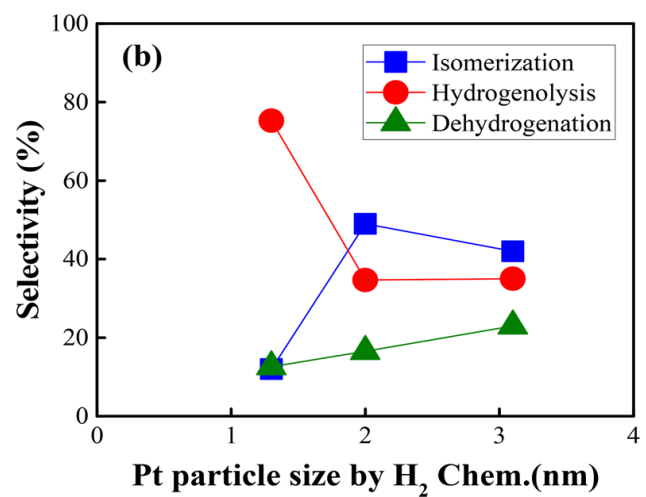

Figure 6. (a) Activity and (b) selectivity extrapolated to zero conversion for isobutane hydrogenolysis and isomerization reaction on different $\mathrm{Pt} / \mathrm{SiO}_{2}$ particle size.

occurred selectively on the Pt monometallic previously covered by irreversibly adsorbed hydrogen.

The SOMC/M refilling approach developed on this basis allowed the partial controlled variation of the particle size by adding one Pt layer with a stepwise particle size increase of around $0.6 \mathrm{~nm}$ on top of the Pt nanoparticle obtained after the first refilling run, as obtained from TEM or $\mathrm{H}_{2}$ chemisorption analysis and then confirmed by DFT calculations. The metal loading could be kept at a very low level $(<1-2 \mathrm{wt} \%)$ relevant for technical applications. The particle size distribution remained relatively narrow even after two refilling runs, 
allowing more precise relationships between particle size and catalytic properties to be established. The $\mathrm{TOR}_{\mathrm{H}}$ (for hydrogenolysis) dramatically decreased, while $\mathrm{TOR}_{\mathrm{I}}$ (for skeletal isomerization) slightly increased with increasing particles size. It was therefore suggested that hydrogenolysis might preferentially occur on low coordination surface platinum atoms (corners and edges), while isomerization occurs mostly on the facets.

\section{ASSOCIATED CONTENT}

\section{S Supporting Information}

The Supporting Information is available free of charge on the ACS Publications website at DOI: 10.1021/acs.jpcc.8b06346.

$\mathrm{UV}$-vis spectra for $\mathrm{Pt}(\mathrm{acac})_{2}$ dissolved in toluene. Total amount of $\mathrm{Pt}$ adsorbed on silica bare surface at different temperatures. $\mathrm{H}_{2}$ chemisorption isotherm at $35{ }^{\circ} \mathrm{C}$ on a series of $\mathrm{Pt} / \mathrm{SiO}_{2}$ catalysts prepared by SOMC. Number of moles of $\mathrm{Pt}$ in the surface during refilling run. Activity and selectivity of $\mathrm{Pt} / \mathrm{SiO}_{2}$ catalysts prepared by SOMC. Activity, selectivity of hydrogenolysis, selectivity of isomerization, and selectivity of dehydrogenation as a function of time over a series of $\mathrm{Pt} / \mathrm{SiO}_{2}$ catalysts prepared by SOMC. Influence of contact time on activity and selectivity of $\mathrm{Pt} / \mathrm{SiO}_{2}$ catalyst prepared by SOMC (PDF)

\section{AUTHOR INFORMATION}

\section{Corresponding Authors}

*E-mail: moussab.harb@kaust.edu.sa (Moussab Harb). Phone: +966 (0)128080788.

*E-mail: jeanmarie.basset@kaust.edu.sa (Jean-Marie Basset). Phone: +966 (0)12 8080299.

\section{ORCID $\odot$}

Moussab Harb: 0000-0001-5540-9792

Samy Ould-Chikh: 0000-0002-3486-0944

Jean-Marie Basset: 0000-0003-3166-8882

\section{Notes}

The authors declare no competing financial interest.

\section{ACKNOWLEDGMENTS}

This work was supported by King Abdullah University of Science and Technology.

\section{REFERENCES}

(1) Gates, B. C. Supported Metal Clusters: Synthesis, Structure, and Catalysis. Chem. Rev. 1995, 95, 511-522.

(2) Zhang, Y.; Kang, D.; Saquing, C.; Aindow, M.; Erkey, C. Supported Platinum Nanoparticles by Supercritical Deposition. Ind. Eng. Chem. Res. 2005, 44, 4161-4164.

(3) Roldan Cuenya, B.; Behafarid, F. Nanocatalysis: Size- and ShapeDependent Chemisorption and Catalytic Reactivity. Surf. Sci. Rep. 2015, 70, 135-187.

(4) Cao, S.; Tao, F.; Tang, Y.; Li, Y.; Yu, J. Size- and ShapeDependent Catalytic Performances of Oxidation and Reduction Reactions on Nanocatalysts. Chem. Soc. Rev. 2016, 45, 4747-4765.

(5) Haruta, M. Size- and Support-Dependency in the Catalysis of Gold. Catal. Today 1997, 36, 153-166.

(6) Bond, G. C.; Wells, P. B. Charactrization of the Standard Platnium/Silica Catalyst Europt-1.2. Preparation, Physical Properties, and Chemical Composition. Appl. Catal. 1985, 18, 225-230.

(7) Frenneti, A.; Wells, P. B. Characterization of the Standard Platinum/Silica Catalyst Europt-1.4. Chemisorption of Hybrogen. Appl. Catal. 1985, 18, 243-257.
(8) Fulop, E.; Gnutzmann, V.; Paal, Z.; Vogel, W. Europt-1 After Various Pretreatments: Structure, Surface Composition and Catalytic Activity in Methylcyclopentane Reactions. Appl. Catal. 1990, 66, 319-335.

(9) Carter, J. L.; Cusumano, J. A.; Sinfelt, J. H. Catalysis over Supported Metals. V. The Effect of Crystalline Size on the Catalytic Activity of Nickel. J. Phys. Chem. 1966, 70, 2257-2263.

(10) Bond, G. C.; Gelsthorpe, M. R. Hydrogenolysis of Alkanes. Part 4. - Hydrogenolysis of Propane, n-Butane and Isobutane over Pt/ $\mathrm{Al}_{2} \mathrm{O}_{3}$ and Pt-Re $/ \mathrm{Al}_{2} \mathrm{O}_{3}$ Catalysts. J. Chem. Soc., Faraday Trans. 1 1989, 85, 3767-3783.

(11) Boudart, M. Catalysis by Supported Metals. Adv. Catal. 1969, $20,153-166$

(12) Sushumna, I.; Ruckenstein, E. Events Observed and Evidence for Crystallite Migration in $\mathrm{Pt} / \mathrm{Al}_{2} \mathrm{O}_{3}$ Catalysts. J. Catal. 1988, 109, 433-462.

(13) Bett, J. A.; Kinoshita, K.; Stonehart, P. Crystallite Growth of Platinum Dispersed on Graphitized Carbon Black. J. Catal. 1974, 35, $307-316$.

(14) Fiedorow, R. M. J.; Chahar, B. S.; Wanke, S. E. The Sintering of Supported Metal Catalysts. J. Catal. 1978, 51, 193-202.

(15) Agnelli, M.; Candy, J.-P.; Basset, J.-M.; Bournonville, J. P.; Ferretti, O. A. Surface Organomettalic Chemistry on Metals: III. Formation of a Bimetallic Ni-Sn Phase Generated by Reaction of a $\mathrm{Sn}\left(n-\mathrm{C}_{4} \mathrm{H}_{9}\right)_{4}$ and Silica-Supported Nickel Oxide. J. Catal. 1990, 121, 236-247.

(16) Chen, M.; Schmidt, L. D. Morphology and Sintering of Pt Crystallites on Amorphous $\mathrm{SiO}_{2}$. J. Catal. 1978, 55, 348-360.

(17) Bentahar, F. Z.; Candy, J.-P.; Basset, J.-M.; Le Peltier, F.; Didillon, B. Surface Organometallic Chemistry on Metals in Water: Chemical Modification of Platinum Catalyst Surface by Reaction With Hydrosoluble organotin complexes: Application to the Selective Dehydrogenation of Isobutane to Isobutene. Catal. Today 2001, 66, 303-308.

(18) Basset, J.-M.; Lefebvre, F.; Santini, C. Surface Organometallic Chemistry: Some Fundamental Features Including the Coordination Effects of the Support. Coord. Chem. Rev. 1998, 178, 1703-1723.

(19) Basset, J.-M.; Candy, J.-P.; Louessard, P.; Ferretti, O. A.; Bournonville, J. P. Some Aspects of Surface Organometallic Chemistry on Metals. Application to Selective Hydrogenolysis of Ethyl Acetate into Ethanol on Supported Rhodium-Tin, RutheniumTin, and Nickel-Tin Catalysts. Wiss. Z. Technol. Hochsch. Carl Schorlemmer Leuna-Merseburg 1990, 32, 657-667.

(20) Yao, H. C.; Shelef, M. Hydrogenolysis of Neopentane and nPentane over a $\mathrm{Rh} \gamma-\mathrm{Al}_{2} \mathrm{O}_{3}$ Catalyst. J. Catal. 1979, 56, 12-20.

(21) Locatelli, F.; Candy, J.-P.; Didillon, B.; Niccolai, G. P.; Uzio, D.; Basset, J.-M. Hydrogenolysis of Cyclohexane over $\mathrm{Ir} / \mathrm{SiO}_{2}$ Catalyst: A Mechanistic Study of Carbon-Carbon Bond Cleavage on Metallic Surfaces. J. Am. Chem. Soc. 2001, 123, 1658-1663.

(22) Benfield, R. E. Mean Coordination Numbers and the NonMetal-Metal Transition in Clusters. J. Chem. Soc., Faraday Trans. 1992, 88, 1107-1110.

(23) Goguet, A.; Aouine, M.; Aires, F. J. C. S.; Mallmann, A. D.; Schweich, D.; Candy, J. P. Preparation of a $\mathrm{Pt} / \mathrm{SiO}_{2}$ Catalyst I. Interaction Between Platinum Tetrammine Hydroxide and the Silica Surface. J. Catal. 2002, 209, 135-144.

(24) Goguet, A.; Schweich, D.; Candy, J. P. Preparation of a Pt/SiO Catalyst II. Temperature-Programmed Decomposition of the Adsorbed Platinum Tetrammine Hydroxide Complex Under Flowing Hydrogen, Oxygen, and Argon. J. Catal. 2003, 220, 280-290.

(25) Santana, R. C.; Jongpatiwut, S.; Alvarez, W. E.; Resasco, D. E. Gas-Phase Kinetic Studies of Tetralin Hydrogenation on Pt/Alumina. Ind. Eng. Chem. Res. 2005, 44, 7928-7934.

(26) Yasuda, H.; Yoshimura, Y. Hydrogenation of Tetralin over Zeolite-Supported Pd-Pt Catalysts in the Presence of Dibenzothiophene. Catal. Lett. 1997, 46, 43-48.

(27) Scholten, J. J. F.; van Montfoort, A. The Determination of the Free-Metal Surface Area of Palladium Catalysts. J. Catal. 1962, 1, 8592. 
(28) Candy, J. P.; et al. Hydrogen Adsorption on Platinum Catalysts: Quantitative Determination of the Various Species Population. J. Chem. Soc., Faraday Trans. 1 1980, 76, 616-629.

(29) Womes, M.; Cholley, T.; Peltier, F. L.; Morin, S.; Didillon, B.; Szydlowski-Schildknecht, N. Study of the Reaction Mechanisms Between $\mathrm{Pt}(\mathrm{acac})_{2}$ and Alumina Surface Sites Application to a New Refilling Technique for the Controlled Variation of the Particle Size of $\mathrm{Pt} / \mathrm{Al}_{2} \mathrm{O}_{3}$ Catalysts. Appl. Catal., A 2005, 283, 9-22.

(30) Candy, J.-P.; El Mansour, A.; Ferretti, O. A.; Mabilon, G.; Bournonville, J. P.; Basset, J.-M.; Martino, G. Surface Organometallic Chemistry on Metals. J. Catal. 1988, 112, 201-209.

(31) Humblot, F.; Candy, J.-P.; Peltier, F. L.; Didillon, B.; Basset, J.M. Surface Organometallic Chemistry on Metals: Selective Dehydrogenation of Isobutane into Isobutene on Bimetallic Catalysts Prepared by Reaction of Tetra n-Butyltin on Silica-Supported Platinum Catalyst. J. Catal. 1998, 179, 459-468.

(32) Humblot, F.; Didillon, D.; Lepeltier, F.; Candy, J.-P.; Corker, J.; Clause, O.; Bayard, F.; Basset, J.-M. Surface Organometallic Chemistry on Metals: Formation of a Stable $\mathrm{tS}_{\mathrm{n}}\left(\mathrm{n}-\mathrm{C}_{4} \mathrm{H}_{9}\right)$ Fragment as a Precursor of Surface Alloy Obtained by Stepwise Hydrogenolysis of $\mathrm{S}_{\mathrm{n}}\left(\mathrm{n}-\mathrm{C}_{4} \mathrm{H}_{9}\right)_{4}$ on a Platinum Particle Supported on Silica. J. Am. Chem. Soc. 1998, 120, 137-146.

(33) Kresse, G.; Hafner, J. Ab-Initio Molecular Dynamics Simulation of the Liquid-Metal-Amorphous-Semiconductor Transition in Germanium. Phys. Rev. B: Condens. Matter Mater. Phys. 1994, 49, 1425114269.

(34) Kresse, G.; Furthmüller, J. Efficient Iterative Schemes for AbInitio Total Energy Calculations Using a Plane-Wave Basis Set. Phys. Rev. B: Condens. Matter Mater. Phys. 1996, 54, 11169-11186.

(35) Kresse, G.; Furthmüller, J. Efficiency of Ab-Initio Total Energy Calculations for Metals and Semiconductors Using a Plane-Wave Basis Set. Comput. Mater. Sci. 1996, 6, 15-50.

(36) Kresse, G.; Joubert, D. From Ultrasoft Pseudopotentials to the Projector Augmented-Wave Method. Phys. Rev. B: Condens. Matter Mater. Phys. 1999, 59, 1758-1775.

(37) Perdew, J. P.; Burke, K.; Ernzerhof, M. Generalized Gradient Approximation Made Simple. Phys. Rev. Lett. 1996, 77, 3865-3868.

(38) Blöchl, P. E. Projector Augmented-Wave Method. Phys. Rev. B: Condens. Matter Mater. Phys. 1994, 50, 17953-17979.

(39) Candy, J.-P.; Didillon, B.; Smith, E. L.; Shay, T. B.; Basset, J.-M. Surface Organomettalic Chemistry on Metals: A Novel and Effetive Route to Custom-Designed Bimetallic Catalysts. J. Mol. Catal. 1994, 86, 179-204.

(40) Datye, A. K. Electron Microscopy of Catalysts: Recent Achievements and Future Prospects. J. Catal. 2003, 216, 144-154.

(41) Cabié, M.; Giorgio, S.; Henry, C. R.; Axet, M. R.; Philippot, K.; Chaudret, B. Direct Observation of the Reversible Changes of the Morphology of Pt Nanoparticles Under Gas Environment. J. Phys. Chem. C 2010, 114, 2160-2163.

(42) de Menorval, L.-C.; Chaqroune, A.; Coq, B.; Figueras, F. Characterization of Mono- and Bi-Metallic Platinum Catalysts Using CO FTIR Spectroscopy Size Effects and Topological Segregation. J. Chem. Soc., Faraday Trans. 1997, 93, 3715-3720.

(43) Blyholder, G. Molecular Orbital View of Chemisorbed Carbon Monoxide. J. Phys. Chem. 1964, 68, 2772-2777.

(44) Blyholder, G.; Allen, M. C. Infrared Spectra and Molecular Orbital Model for Carbon Monoxide Adsorbed on Metals. J. Am. Chem. Soc. 1969, 91, 3158-3162.

(45) Primet, M.; Basset, J.-M.; Mathieu, M. V.; Prettre, M. Infrared Study of $\mathrm{CO}$ Adsorbed on $\mathrm{Pt} / \mathrm{Al}_{2} \mathrm{O}_{3}$. A Method for Determining Metal-Adsorbate Interactions. J. Catal. 1973, 29, 213-223.

(46) Brandt, R. K.; Hughes, M. R.; Bourget, L. P.; Truszkowska, K.; Greenler, R. G. The Interpretation of CO Adsorbed on $\mathrm{Pt} / \mathrm{SiO}_{2}$ of Two Different Particle-Size Distributions. Surf. Sci. 1993, 286, 15-25.

(47) Kappers, M. J.; van der Maas, J. H. Correlation Between CO Frequency and Pt Coordination Number. A DRIFT Study on Supported Pt Catalysts. Catal. Lett. 1991, 10, 365-373.
(48) Stoop, F.; Toolenaar, F. J. C. M.; Ponec, V. Geometric and Ligand Effects in the Infrared Spectra of Adsorbed Carbon Monoxide. J. Catal. 1982, 73, 50-56.

(49) Bartók, M.; Sárkány, J.; Sitkei, A. Investigation of Interactions Between Metals and Adsorbed Organic Compounds by Infrared Spectroscopic Study of Adsorbed CO. J. Catal. 1981, 72, 236-245.

(50) Primet, M. Electronic Transfer and Ligand Effects in the Infrared Spectra of Adsorbed Carbon Monoxide. J. Catal. 1984, 88, 273-282.

(51) Almana, N.; Phivilay, S. P.; Laveille, P.; Hedhili, M. N.; Fornasiero, P.; Takanabe, K.; Basset, J.-M. Design of a Core-Shell $\mathrm{Pt}-\mathrm{SiO}_{2}$ Catalyst in a Reverse Microemulsion System: Distinctive Kinetics on CO Oxidation at Low Temperature. J. Catal. 2016, 340, $368-375$.

(52) Allian, A. D.; Takanabe, K.; Fujdala, K. L.; Hao, X.; Truex, T. J.; Cai, J.; Buda, C.; Neurock, M.; Iglesia, E. Chemisorption of CO and Mechanism of CO Oxidation on Supported Platinum Nanoclusters. J. Am. Chem. Soc. 2011, 133, 4498-4517.

(53) Acres, G. J. K. A. Technique for the Continuous Performance of Liquid-Phase Catalysed Reactions. Platinum Metals Rev. 1967, 11, $86-91$. 